\title{
Structural Transformations of Incoming Signal by a Single Nonlinear Oscillatory Neuron or by an Artificial Nonlinear Neural Network
}

\author{
Roman Peleshchak \\ University of Information Technology and Management, Rzeszow, 35-225, Poland \\ E-mail: rpeleshchak@ukr.net
}

Vasyl Lytvyn

Department of Information Systems and Networks, Lviv Polytechnic National University, Lviv, 79012, Ukraine

E-mail: vasyl.v.lytvyn@lpnu.ua

\author{
Oksana Bihun \\ Department of Mathematics, University of Colorado, Colorado Springs, 80918, USA \\ E-mail: obihun@uccs.edu
}

Ivan Peleshchak

Department of Information Systems and Networks, Lviv Polytechnic National University, Lviv, 79012, Ukraine

E-mail: peleshchakivan@gmail.com

Received: 02 February 2019; Accepted: 20 March 2019; Published: 08 August 2019

\begin{abstract}
Structural transformations of incoming informational signal by a single nonlinear oscillatory neuron or an artificial nonlinear neural network are investigated. The neurons are modeled as threshold devices so that the artificial nonlinear neural network under consideration are systems of nonlinear van der Pol type oscillatory neurons. The neurons are coupled by synaptic weight coefficients to endow the systems with the configuration topology of a chain or a ring. It is shown that the morphology of the outgoing signal - with respect to the shape, amplitude and time dependence of the instantaneous frequency of the signal - at the output of such a neural network has a higher degree of stochasticity than the morphology of the signal at the output of a single neuron. We conclude that the process of coding by a single neuron or an entire chain-like or circular neural network may be considered in terms of frequency modulations, which are known in Physics as a way to transmit information. We conjecture that frequency modulations constitute one of the ways of coding of information by the neurons in these types of neural networks.
\end{abstract}

Index Terms-Nonlinear neuron, artificial nonlinear neural network, coding of information.

\section{INTRODUCTION}

Construction and implementation of mathematical models that yield a high degree of protection of information are among present challenges of cryptography. Models that feature significant changes of the structure of the incoming informational signal are of special interest. In this paper, we study transformations of an incoming signal by a single oscillatory neuron as well as by a system of nonlinear oscillatory neurons coupled by synaptic weight coefficients. These coefficients are chosen to endow the artificial nonlinear neural network with the topology of a chain or a ring. The interaction between the neurons is assumed to be of "dipole dipole" type. We show that the proposed models change the structure (shape, amplitude, frequency and phase) of an incoming informational signal in a significant way.

The structure of the article consists of 5 sections:

- The first section (introduction) indicates the need to build and implement mathematical models based on neural networks with nonlinear oscillatory neurons for cryptographic systems.

- The second section presents an analysis of scientific papers related to the transformation of the structure of input signals by a single neuron or neural network.

- The third section describes the purpose and method of studying the processes of morphology transformation of the input signal using a chain or ring network of nonlinear oscillatory neurons.

- In the fourth section, a mathematical model of the chain and ring network of nonlinear oscillatory neurons is constructed.

- The fifth section presents the solution of the mathematical model of the chain and ring network 
of nonlinear oscillatory neurons and a computer experiment to transform the morphology of the input signals by a single neuron and a system of neurons. The results of studies of the criteria for the occurrence of resonance effects in a nonlinear oscillatory neuron are also presented. It is shown that resonance effects in a nonlinear oscillatory neuron occur under the condition that the frequency of the external non-stationary signal coincides with the intrinsic dynamics of the neuron The coding of information on the basis of frequency modulation using a nonlinear oscillatory neuron is proposed. Decoding using the inverse operator, which acts on the vector of the output signal, is proposed.

\section{RELATED WORKS}

At the initial stages of processing of sensory data, wavelet analysis is an effective instrument in the determination of the informative component in the neural signals that are being registered. Usually this determination is performed by analysis of the structure of the point-wise processes, that is, analysis of the timefrequency dynamics of neural responses [1 - 8], in which the information is carried by the times at which impulses (spikes) are generated rather than the shape of these responses [9]. The mechanisms by which the spikes are generated are only partially understood [10], while the ways in which nonlinear oscillatory neurons transform the structure of an incoming information signal have not been investigated, to the best of our knowledge.

In $[2-5,8]$ the transformation of signals by a sensory neuron (threshold device) is analyzed; the analysis does not take into account the dynamics of the neuron itself. It has been shown, by means of classical models of threshold systems such as "integrate-and-fire" [2 - 4] and "threshold crossing" [5,8], that different characteristics of complex dynamics at the input of a sensory neuron are preserved in the structure of certain pointwise processes [4-8], [11-14].

Numerical modeling of the dynamics of a neuron subject to a stationary external signal, without consideration of the threshold effect of the neuron, was performed in [15]. In contrast, in this paper we consider a model with nonstationary external signals, which are transformed by an artificial nonlinear neural network modeled by a system of nonlinear oscillatory neurons of van der Pole type. In this system, the neural connections are represented by synaptic weight coefficients that take into account the threshold effect of the neuron.

Using the technique of double wavelet analysis, the authors of [16, 17] investigate time-frequency dynamics of the sensory neuron (threshold device), while taking into account both its own dynamics as well as the dynamics generated by the action of a non-stationary external signal. The sensory neuron is modeled in [16,17] as a threshold device that transforms an incoming signal into a sequence of output impulses. This sequence of impulses was described by a sequence of Dirac delta- functions, each function corresponding to the moment of generation of an impulse (spike). These model impulses have the same form and amplitude, thus the information about the external action of the dynamic signal is expressed only by the time intervals between the moments of the generations of the impulses.

The study of structural transformation of incoming signal by a nonlinear oscillatory neuron that has its own dynamics, as well as by an artificial nonlinear neural network that consists of such neurons, contributes to understanding of neural networks of human brain. Indeed, biological neurons of the brain generate waves of various frequencies [18 - 20], which are transmitted as incoming signals to other neurons. It is therefore important to understand the response mechanisms of the latter neurons, that is, hoe the outgoing signals are generated. The proposed study can also be applied to development of coding algorithms that feature high degree of protection of information as well as to recognition of multispectral images.

\section{SETting OBJECTIVES}

In this paper we investigate, by means of an analyticalnumerical method, the processes of transformation of the structure of the incoming signal by a single oscillatory neuron, taking into account its threshold effect, as well as an artificial nonlinear neural network represented by a system of neurons that are van der Pol type oscillators coupled by synaptic weight coefficients. The weight coefficients are chosen to endow the neural networks with the configurational topology of either a chain or a ring.

\section{MATHEMATICAL MODEL}

We consider decoupled as well as coupled systems of nonlinear oscillatory neurons.

In the case of a system of $N$ decoupled neurons, the $k-t h$ sensory nonlinear oscillatory neuron $X_{k}(t)$ is of van der Pol type - a threshold device - such that its own dynamics is described by the parameters $\mu_{k}>0, \omega_{0 k}$ (the own frequency of the $k-t h$ neuron) as well as $p_{k}$ (the amplitude parameter of the $k-t h$ neuron), see Fig. 1. The system reads

$$
\ddot{X}_{k}+\mu_{k}\left[X_{k}^{2}-p_{k}^{2}\left(N_{0 k} ; N_{c k}\right)\right] \dot{X}_{k}+\omega_{0 k}^{2} X_{k}=V_{k}(t)
$$

where $p_{k}^{2}\left(N_{0 k} ; N_{c k}\right)=p_{0 k}^{2} \tanh \left(\frac{N_{0 k}-N_{c k}}{\sigma_{k}^{2}}\right)$ with $N_{0 k}, N_{c k}$ and $\sigma_{k}^{2}$ being the number of the incoming impulses, the threshold number of impulses of the $k-t h$ neuron and the dispersion, respectively, $V_{1}(t)$ is the external incoming informational signal, while for each $k=2,3, \ldots, N$ 


$$
V_{k}(t)=X_{k-1}(t)
$$

is the incoming non-stationary signal on the $k$-th oscillatory neuron. Note that $V_{k}(t)$ equals $X_{k-1}(t)$, the latter being the result of transformation of the external signal $V_{1}(t)$ by $k-1$ previous neurons.

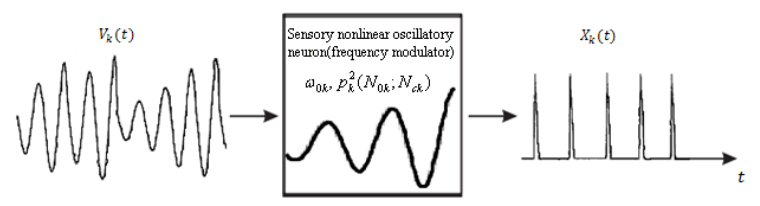

Fig.1. Schematic representation of the process of transformation of an incoming signal $V_{k}(t)$ by a sensory nonlinear oscillatory neuron (threshold device). The moments of time at which impulses are generated at the output of the threshold device $X_{k}(t)$ correspond to the moments of time at which the threshold level is crossed.

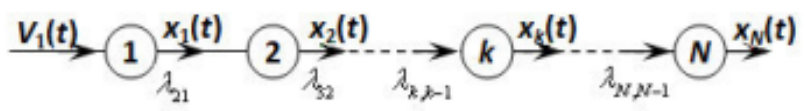

Fig.2. Schematic representation of an artificial nonlinear neural network with the topology of a chain and one-sided connections manifested by weight coefficients $\lambda_{k, k-1}$, where $k=1,2, \ldots, N$.

Note that $\lambda_{N 1}=0$.
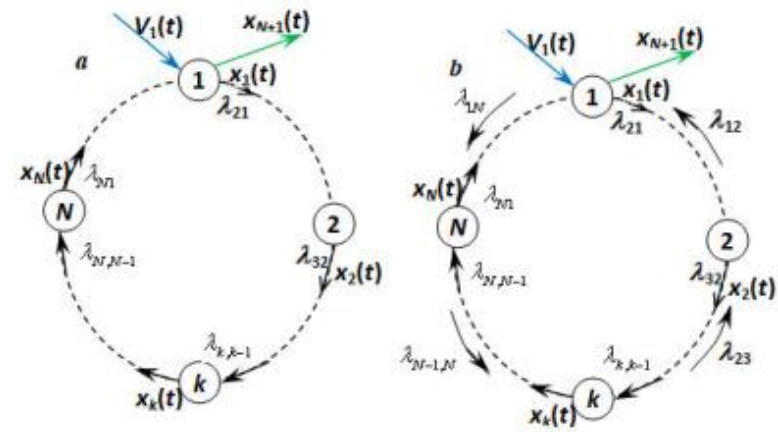

Fig.3. Schematic representation of an artificial nonlinear neural network with the topology of a chain and (a) one-sided connections manifested by weight coefficients $\lambda_{k, k-1}$ and $\lambda_{N 1}$ or (b) two-sided connections manifested by weight coefficients $\lambda_{k, k-1}, \lambda_{k-1, k}, \lambda_{N 1}$ and $\lambda_{1 N}$, where

$$
k=1,2, \ldots, N \text {. }
$$

In the case of a coupled system of $N$ nonlinear oscillatory neurons, we assume that the neurons are related among themselves by synaptic weight coefficients $\lambda_{j k}$, with the topology of a chain (Fig. 2) or a ring (Fig. 3). This artificial nonlinear neural network is described by a system of nonlinear differential equations

$$
\ddot{X}_{k}+\mu_{k}\left\{\left[X_{k}+\sum_{j=1}^{N} \lambda_{j k} X_{j}\right]^{2}-p_{k}^{2}\left(N_{0 k} ; N_{c k}\right)\right\} \dot{X}_{k}+\omega_{0 k}^{2}\left[X_{k}+\sum_{j=1}^{N} \lambda_{j k} X_{j}\right]=V_{k}(t)
$$

where the "dot" denotes differentiation with respect to the time-variable $t, k=1,2, \ldots, N$ and all the other functions and parameters of the system are defined as above. We assume that $\lambda_{j j}=0$. The desired topology of the artificial nonlinear neural network is achieved by an appropriate choice of the weight coefficients. For example, if $\lambda_{j k}=0$ for all $j, k$ such that $j \neq k+1$, where $\lambda_{N, N+1}=\lambda_{N 1}$, the $k$-th equation of system (3) involves only the dependent variables $X_{k}$ and $X_{k+1}$, where $X_{N+1}$ is identified with $X_{1}$, so that the neural network has the topology of a ring, see Fig. 3(a). If, in addition, $\lambda_{N 1}=0$, the ring is broken, and the network has the topology of a chain, see Fig. 2. In the case where $\lambda_{j k}=0$ for all $j, k$ that satisfy $j \notin\{k-1, k+1\}$, the system has the topology of a ring, with two-sided links between consecutive neurons, see Fig. 3(b).

In both cases, the nonlinear oscillatory neurons $X_{k}$ in systems (1) and (3) can generate impulses even if the external non-stationary signal is absent $\left(V_{1}(t) \equiv 0\right)$, provided that the number of the impulses $N_{0 k}$ present in the sensory neuron exceeds the threshold value $N_{c k}$, that is, $N_{0 k}>N_{c k}$. Indeed, in this case, $p_{k}^{2}\left(N_{0 k}, N_{c k}\right)>0$ [18] Therefore, each nonlinear neuron $X_{k}$ can be considered as a threshold device that transforms the incoming nonstationary signal $V_{k}(t)$ (which arrives at the $k-t h$ neuron) into a sequence of impulses at the output (see Fig. 1). This effect is due to the overlay of the dynamics of the incoming non-stationary signal with the own dynamics of the neuron. Due to the latter dynamics, the structure of the signal at the output of the $k-t h$ nonlinear neuron is significantly changed in comparison with the incoming signal $V_{k}(t)$.The complex dynamics of the transformation of the incoming non-stationary signal is discussed in [18].

Possible applications of neural network technologies include detection of multispectral images, detection and recognition of computer attacks, compression of input images [21-23].

In the next section, a three-neuron system that models an artificial nonlinear neural network with ring-like topology is studied. The Krylov-BogolyubovMytropolsky method of subsequent approximations utilized for numerical solution of this system is summarized. Several incoming informational signals are considered: a single rectangular impulse, a superposition of several rectangular impulses, a superposition of products of periodic signals times a Gaussian functions. Plots of the output informational signal and of the frequency modulation of the incoming signal due to its modification by the appropriate artificial nonlinear neural network are provided. 


\section{ENCODING VIA AN ARTIFICIAL NONLINEAR NEURAL NETWORK WITH ONE OR THREE VAN DER POL TYPE OSCILLATORY NEURONS}

In this section we consider a coupled system of 3 oscillatory neurons described by system (3) with $N=3$ with chain-like topology (see Fig. 2), which reads

$$
\begin{gathered}
\ddot{X}_{1}+\mu_{1}\left[\tilde{X}_{1}^{2}-p_{1}^{2}\left(N_{01} ; N_{c 1}\right)\right] \dot{X}_{1}+\omega_{01}^{2} \tilde{X}_{1}=V_{1}(t) \\
\ddot{X}_{2}+\mu_{2}\left[\tilde{X}_{2}^{2}-p_{2}^{2}\left(N_{02} ; N_{c 2}\right)\right] \dot{X}_{2}+\omega_{02}^{2} \tilde{X}_{2}=X_{1}(t) \\
\ddot{X}_{3}+\mu_{3}\left[\tilde{X}_{3}^{2}-p_{3}^{2}\left(N_{03} ; N_{c 3}\right)\right] \dot{X}_{3}+\omega_{03}^{2} \tilde{X}_{3}=X_{2}(t)
\end{gathered}
$$

where

$$
\begin{gathered}
\tilde{X}_{1}=X_{1}+\lambda_{21} X_{2} \\
\tilde{X}_{2}=X_{2}+\lambda_{12} X_{1}+\lambda_{32} X_{3} \\
\tilde{X}_{3}=X_{3}+\lambda_{23} X_{2}
\end{gathered}
$$

We solve system (4) by Krylov-BogolyubovMytropolsky [24] method of iterative approximations. Below we summarize the method for the case of the decoupled system (1), for the sake of clarity. The application of the method to system (4) is done by an analogous procedure.

In system (1), we set

$$
\begin{gathered}
X_{k}^{(n)}(t)=a_{k}^{(n)}(t) \sin \psi_{k}^{(n)}(t) \\
\psi_{k}^{(n)}(t)=\omega_{0 k} t+\phi_{k}^{(n)}(t)
\end{gathered}
$$

where $n=1,2, \ldots$ is the iteration number and $a_{k}^{(n)}(t)$, $\phi_{k}^{(n)}(t)$ are functions of the time variable $t$ chosen so that (6) satisfies the $k-t h$ equation in system (1). We require that $a_{k}(t)$ is a "slow-changing" function, that is,

$$
\lim _{t \rightarrow \infty} \frac{a_{k}^{(n)}\left(p_{k} \mu_{k} t\right)}{a_{k}^{(n)}\left(p_{k} t\right)}=1
$$

Because substitution of (6) into the $k-$ th equation of system (1) results with one differential equation for two unknown functions $a_{k}^{(n)}(t)$ and $\psi_{k}^{(n)}(t)$, we impose an additional condition

$$
\dot{X}_{k}^{(n)}=\omega_{0 k} a_{k}^{(n)}(t) \cos \psi_{k}^{(n)}(t)
$$

which is equivalent to

$$
\dot{a}_{k}^{(n)}(t) \sin \psi_{k}^{(n)}(t)+a_{k}^{(n)}(t) \dot{\psi}_{k}^{(n)}(t) \cos \psi_{k}^{(n)}(t)-a \omega \cos \psi_{k}^{(n)}(t)=0 .
$$

Upon substitution of (5) into the $k-t h$ equation of system (1), taking into account condition (10), applying an iterative procedure idea, we obtain the following iterative process for determining $a_{k}^{(n)}(t)$ and $\psi_{k}^{(n)}(t)$ :

$$
\begin{aligned}
& \frac{d a_{k}^{(n)}}{d t}=-a_{k}^{(n-1)} \mu_{k}\left[\left(a_{k}^{(n-1)}\right)^{2} \sin ^{2} \psi_{k}^{(n-1)}-p_{k}^{2}\right] \cos ^{2} \psi_{k}^{(n-1)}+\frac{1}{\omega_{0 k}} V_{k} \cos \psi_{k}^{(n-1)} \\
& \frac{d \psi_{k}^{(n)}}{d t}=\omega_{0 k}+\mu_{k}\left[\left(a_{k}^{(n-1)}\right)^{2} \sin ^{2} \psi_{k}^{(n-1)}-p_{k}^{2}\right] \sin \psi_{k}^{(n-1)} \cos \psi_{k}^{(n-1)}-\frac{1}{\omega_{0 k}\left(a_{k}^{(n-1)}\right.} V_{k} \sin \psi_{k}^{(n-1)}
\end{aligned}
$$

where $n=1,2, \ldots$ and $p_{k}^{2}=p_{k}^{2}\left(N_{0 k}, N_{c k}\right)$ as in (1).

Let us discuss the choice of the initial approximations $a_{k}^{(0)}(t)$ and $\psi_{k}^{(0)}(t)$ in the iterative process (11). Take an average of the right-hand side of system (11) with respect to the interval $[0,2 \pi]$ using the rule from [17]:

$$
<\Phi>=\frac{1}{2 \pi} \int_{0}^{2 \pi} \Phi\left(\psi_{k}^{(n)}\right) d \psi_{k}^{(n)}
$$

For the initial (zeroth) approximation, the expressions for the functions $a_{k}^{(0)}(t)$ and $\psi_{k}^{(0)}(t)$ are found from the following decoupled system of equations:

$$
\begin{gathered}
\frac{d a_{k}^{(0)}}{d t}=-a_{k}^{(0)} \mu_{k}\left(\frac{\left(a_{k}^{(0)}\right)^{2}}{8}-\frac{p_{k}^{2}}{2}\right) \\
\frac{d \psi_{k}^{(0)}}{d t}=\omega_{0 k} .
\end{gathered}
$$

The following two particular solutions of the last system (14) are chosen as the initial approximations in the iterative process (10):

$$
\begin{gathered}
a_{k}^{(0)}(t)=\frac{2 p_{k}}{\sqrt{1+e^{-p_{k}^{2} \mu_{k} t}}} \\
\psi_{k}^{(0)}(t)=\omega_{0 k} t
\end{gathered}
$$

where the function $a_{k}^{(0)}(t)$ satisfies the criterion (8) of slow changing function. To find expressions for $a_{k}^{(1)}(t)$ and $\psi_{k}^{(1)}(t)$ in the first approximation $(n=1)$, expressions (15), (16) are inserted into system (11). Upon integration, the approximations $a_{k}^{(1)}(t)$ and $\psi_{k}^{(1)}(t)$ are obtained. And so on. The iteration process terminates if the following conditions are satisfied:

$$
\left|\frac{a_{k}^{(\mathrm{n})}(t)-a_{k}^{(\mathrm{n}-1)}(t)}{a_{k}^{(\mathrm{n})}(t)}\right|<\delta,\left|\frac{\psi_{k}^{(\mathrm{n})}(t)-\psi_{k}^{(\mathrm{n}-1)}(t)}{\psi_{k}^{(\mathrm{n})}(t)}\right|<\delta
$$

for all $k=1,2, \ldots, N$ and for all $t$ from the interval on which the solution of system (1) is sought, where $\delta$ is the desired accuracy (in the subsequent examples, $\left.\delta=10^{-5}\right)$. 
System (4) of three nonlinear differential equations is solved by a similar procedure using the KrylovBogolyubov-Mytropolsky method [24] and the method of subsequent approximations. More precisely, at the initial step system (4) is solved without taking into account the synaptic weight coefficients $\lambda_{j k}$. The solutions of this special case of system (4) are then set to be the initial approximation of the solution of (the most general form of) system (4). In the subsequent iterations, the solutions of the preceding approximation are inserted into the summands that contain the weight synaptic coefficients of system (4). Once conditions (17) are satisfied, the iteration process is terminated and $X_{k}^{(n)}(t)$ given by (6) is accepted as an approximate solution of system (4).

As an example, we choose the external non-stationary signal $V_{1}(t)$ o be the sum of $L$ simple non-stationary signals, each centered at the point $t=t_{\ell}$ and characterized by the system of parameters $M_{\ell}$, see (21). More precisely,

$$
V_{1}(t)=\sum_{\ell=0}^{L-1} \tilde{\lambda}_{\ell 1} v_{\ell 1}\left(t-t_{\ell}\right)+\tilde{\lambda}_{01}^{*}
$$

where the functions $v_{\ell 1}$ are the incoming simple signals. Each among the latter functions may be chosen to be, for example, a product of a Gaussian curve and an oscillatory function:

$$
v_{\ell 1}\left(t-t_{\ell}\right)=\frac{1}{2 \tau_{\ell} \sqrt{\pi}} \exp \left[-\frac{\left(t-t_{\ell}\right)^{2}}{4 \tau_{\ell}^{2}}\right] \cos \left(\tilde{\omega}_{\ell}\left(t-t_{\ell}\right)+\alpha_{\ell}\right)
$$

The non stationary signal $V_{1}(t)$ given by (18) and (19) is described by the system of parameters

$$
\begin{gathered}
M_{0}=\left(\lambda_{01}^{*}\right) \\
M_{\ell}=\left(\tilde{\lambda}_{\ell 1}, \tilde{\omega}_{\ell}, t_{\ell}, \tau_{\ell}, \alpha_{\ell}\right), 1 \leq \ell \leq L,
\end{gathered}
$$

where each $\tilde{\lambda}_{\ell 1}$ is the weight coefficient representing the connection of the simple signal $v_{\ell 1}$ with the neuron $X_{1}(t) ; \tilde{\lambda}_{01}$ is the weight coefficient of the signal shift; $\omega_{\ell}$ is the external frequency of the oscillations that bear the information in the incoming signal, in $\mathrm{Hz} ; t_{\ell}$ is the center of localization of the signal with respect to time, in seconds; $\tau_{\ell}$ is the length of the characteristic time interval of the signal localization, in seconds; $\alpha_{\ell}$ is the initial phase, in radians.

Formula (18) describes the superposition of the incoming signals (19) that arrive with the weight coefficients $\tilde{\lambda}_{\ell 1}$ at the input of the first neuron (incoming operator), with the the shift signal $\lambda_{01}^{*}$. The input sum operator $\left[f_{i n}\right]_{1}$ transforms the incoming signals weighed by the coefficients $\tilde{\lambda}_{\ell 1}$ and feeds them into the activation operator $\left[f_{a}\right]_{1}$, see Fig. 4 . In the case of decoupled system (1), for the $k-t h$ sensory nonlinear oscillatory neuron, the activation operator has the following form:

$$
\left[f_{a}\right]_{k}=\frac{d^{2}}{d t^{2}}+\mu_{k}\left[X_{k}^{2}-p_{k}^{2}\left(N_{0 k} ; N_{c k}\right)\right] \frac{d}{d t}+\omega_{0 k}^{2}
$$

where $k=1,2, \ldots, N$.

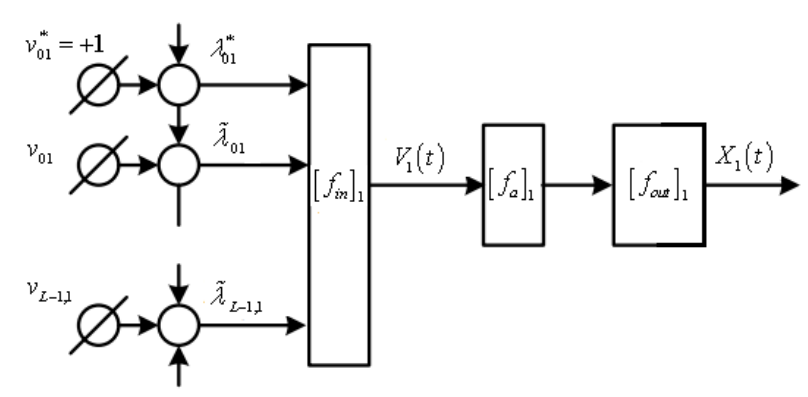

Fig.4. The structure of artificial nonlinear oscillator neuron (coding scheme).

The output signal of the $k-t h$ nonlinear oscillatory neuron, see Fig. 4, is the output signal of the output operator $\left[f_{\text {out }}\right]_{k}$, for which the input is the output of the activation operator $\left[f_{a}\right]_{k}$. The output operator $\left[f_{\text {out }}\right]_{k}$ is necessary for the representation of the state of the neuron by values within a desired range. In the majority of literature this last operator is not emphasized and the output signal of the neuron is assumed to be the output of the activation operator $\left[f_{a}\right]_{k}$. However, for the purpose of analysis and synthesis of artificial neural networks that have different activation functions with different domains and ranges, it is necessary to take the output operator $\left[f_{\text {out }}\right]_{k}$ into account. Therefore, the nonlinear operator of transformation of the vector of incoming signals into the vector of the output signal can be written in the following form:

$$
\begin{gathered}
X_{1}(t)=\left[f_{\text {out }}\right]_{1}\left(\left[f_{a}\right]_{1}\left(\left[f_{\text {in }}\right]_{1}(v, \tilde{\lambda})\right)\right) \\
X_{k}(t)=\left[f_{\text {out }}\right]_{k}\left(\left[f_{a}\right]_{k}\left(\left[f_{\text {in }}\right]_{k}\left(X_{k-1}(t)\right)\right)\right), k=2,3, \ldots, N
\end{gathered}
$$

where $v=\left(v_{01}, \ldots, v_{L 1}\right)$ and $\tilde{\lambda}=\left(\tilde{\lambda}_{01}, \ldots, \tilde{\lambda}_{L 1}\right)$.

By choosing different values of the parameters $M_{\ell}$, see (21), one can construct a theoretical model that adequately represents real physical (or biological) processes, which occur as the external non-stationary 
signal interacts with the own dynamics of the physical (biological) nonlinear neuron.

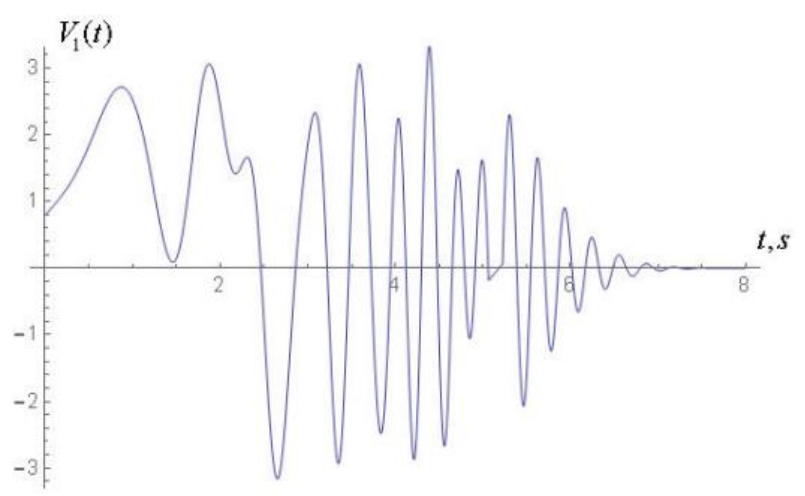

Fig.5. The morphology of the external non-stationary signal $V_{1}(t)$ that is a superposition of five simple signals. The signal $V_{1}(t)$ is given by (18), (19) with $L=4$, the weight coefficients $\lambda_{01}=\lambda_{\ell 1}=4, \lambda_{01}^{*}=0$, the centers of localization of the simple signals $t_{\ell}=1+\ell$, the lengths of time-intervals of localization $\tau_{\ell}=0,5 \mathrm{~s}$ and the frequencies $\tilde{\omega}_{\ell}=\frac{8 \ell}{5}$, and the initial phases $\alpha_{\ell}=0, \quad \ell=1,2,3,4$.

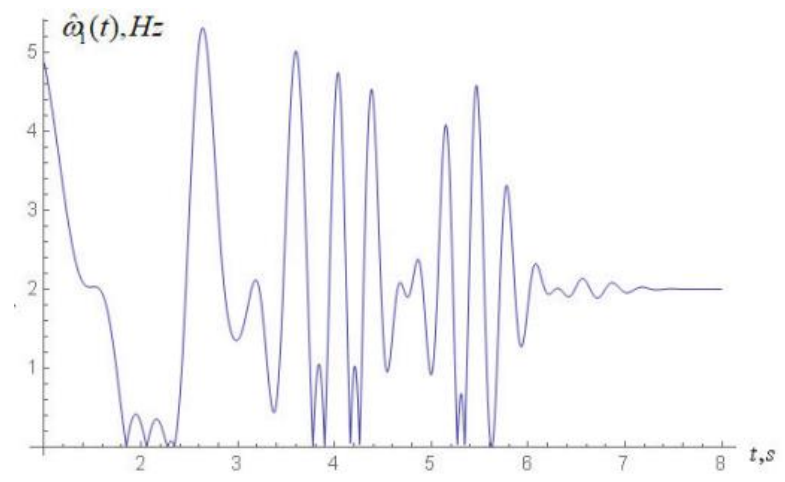

Fig.6. Time dependence of the instantaneous frequency $\tilde{\omega}_{1}(t)$ of the bearing informational signal $V_{1}(t)$ plotted in Fig. 5; $\tilde{\omega}_{1}(t)=\frac{d \psi_{1}^{(1)}(t)}{d t}$ is given by the second equation of system (11).

Fig. 5 shows the graph of the external non-stationary signal $V_{1}(t)$ with $L=4$. This signal is a superposition of five simple non-stationary signals $v_{01}=+1$ and $v_{\ell 1}$, $\ell=1,2,3,4$ given by (19), each arriving at the first sensory oscillatory neuron with the same weight coefficient $\lambda_{\ell 1}=4$ of connection with the first neuron and with the same length of the time-interval of localization given by $\tau_{\ell}=0,5 \mathrm{~s}$. The peak of the Gaussian curve in each signal $v_{\ell 1}$ is chosen to be at $t_{\ell}=\ell+1$. The frequencies $\tilde{\omega}_{\ell}$ of each simple non stationary signal $v_{\ell 1}\left(t-t_{\ell}\right)$ increase linearly with respect to the index $\ell$ so that $\tilde{\omega}_{\ell}=\frac{4 \ell}{5}$. This graph, together with the frequency modulation graph in Fig. 6, shows the moments of time at which local frequencies arise and represents the transitional stages of the transformation of the frequency spectrum of the signal. The instantaneous frequency $\tilde{\omega}_{1}(t)$ of the informational signal $V_{1}(t)$ is given by $\tilde{\omega}_{1}(t)=\frac{d \psi_{1}^{(1)}(t)}{d t}$; it is given by the second equation of system (11).

The comparison of the morphology of the informational signal in Fig. 5 at the input of the first nonlinear oscillatory neuron with the morphology at the output of the same neuron, see Fig. 7, shows that the structure of the incoming signal $V_{1}(t)$ is changed significantly due to the interaction of the internal dynamics of the neuron with the external signal $V_{1}(t)$.

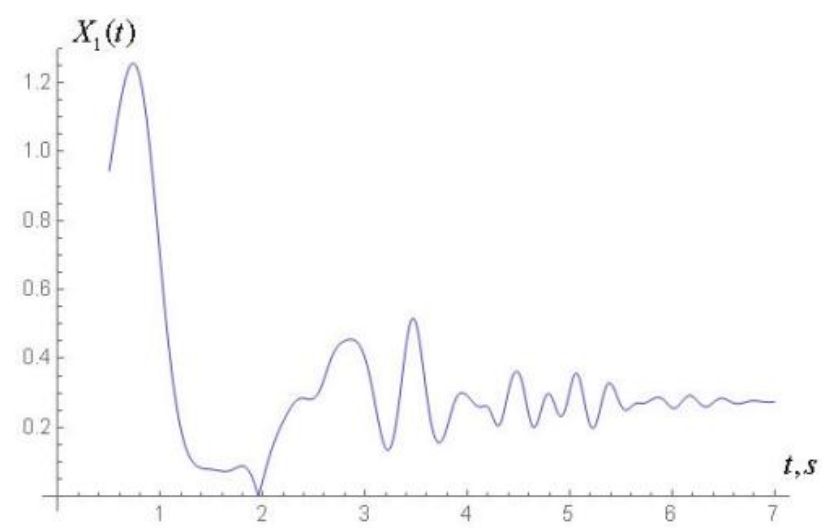

Fig.7. The morphology of the signal at the output of the first nonlinear oscillatory neuron $X_{1}(t)$, the input signal being $V_{1}(t)$ plotted in Fig. 5;

$X_{1}(t)$ is the solution of system (1) with $N=1$ and the parameters $p_{1}=0,4, \mu_{1}=0,1$ and $\omega_{01}=2$.

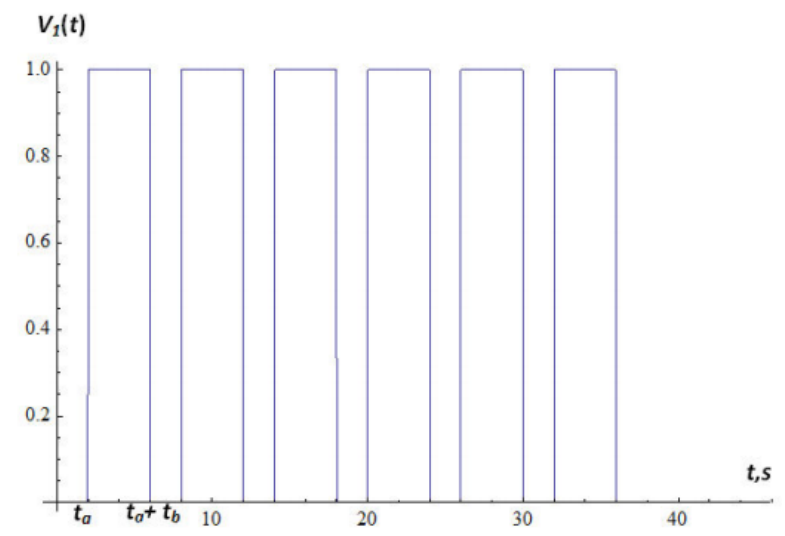

Fig.8. The bearing incoming information signal $V_{1}(t)$ in the form of a superposition of rectangular impulses, each of the width $4 s$; the period $T=6 s$. The signal $V_{1}(t)$ is given by (25) with $L=6, \lambda_{\ell 1}=1$ for all

$\ell=0,1, \ldots, 15 ; \quad t_{a}=2$ and $t_{b}=4$; it arrives at the first nonlinear oscillatory neuron.

The graphs of the external non-stationary signal $V_{1}(t)$ in the form of a superposition of rectangular impulses and a single rectangular impulse are presented in Fig. 8, 9. Recall that the signal $V_{1}(t)$ arrives at the input of the first nonlinear oscillatory neuron. The signal plotted in Fig. 8 is given by 
$V_{1}(t)=\sum_{\ell=0}^{L-1} \tilde{\lambda}_{\ell 1}\left\{\theta\left[t-\left((\ell+1) t_{a}+\ell t_{b}\right)\right]-\theta\left[t-(\ell+1)\left(t_{a}+t_{b}\right)\right]\right\} v_{\ell 1}\left(t-t_{\ell}\right)+\tilde{\lambda}_{01}^{*}$

where $\theta$ is the Heaviside step function defined by $\theta(t)=0$ if $t<0$ and $\theta(t)=1$ if $t \geq 0$.

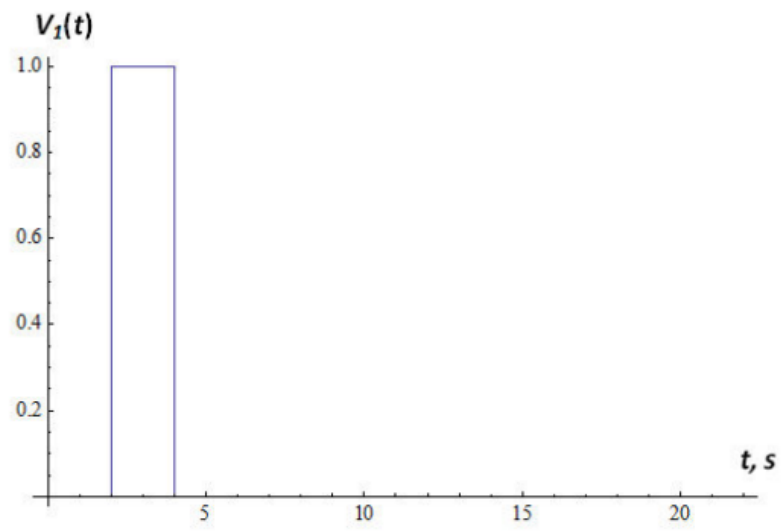

Fig.9. Bearing incoming information signal in the form of a single rectangle, with the impulse width of $2 s$. The signal $V_{1}(t)$ is given by (25)

with $L=1, \lambda_{11}=1, t_{a}=t_{b}=2$; it arrives at the first nonlinear oscillatory neuron.

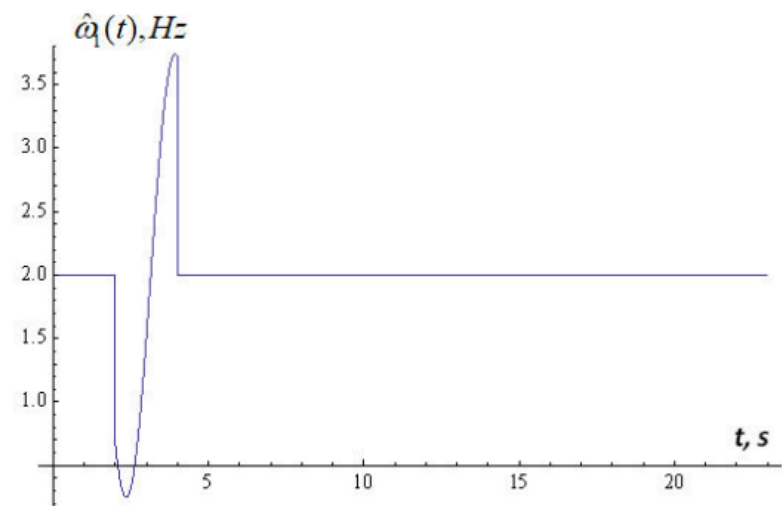

Fig.10. Frequency modulation $\hat{\omega}_{1}(t)$ of the signal $V_{1}(t)$ plotted in Fig. 9.

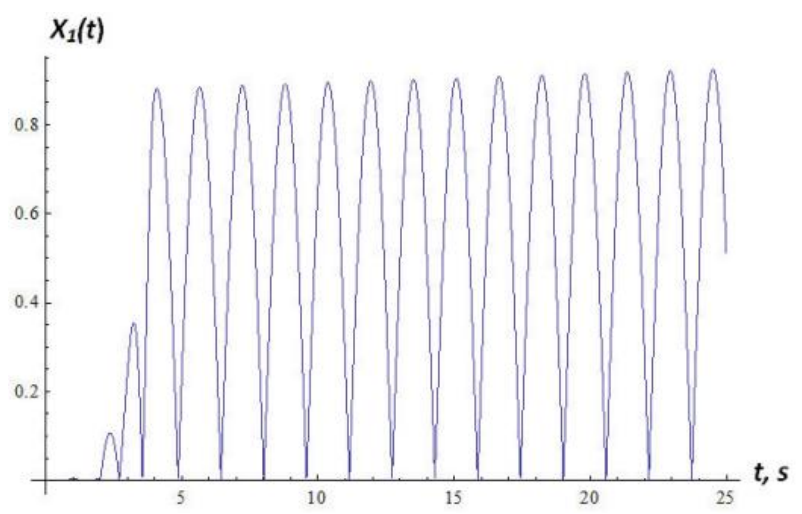

Fig.11. Morphology of the signal $X_{1}(t)$ at the output of the first oscillatory neuron, if the input signal $V_{1}(t)$ is the rectangular impulse plotted in Fig. 9. The signal $X_{1}(t)$ is the solution of system (1) with $N=1$ and the parameters $p_{1}=0,4, \mu_{1}=0,1$ and $\omega_{01}=2$.
Fig. 10 provides the graph of the frequency modulation of the external signal $V_{1}(t)$ plotted in Fig. 9. This graph shows the moments at which local frequencies arise as well as the transitional stages of the transformation of the frequency spectrum of the signal.

Fig. 12 shows the morphology of the signal at the output of a single nonlinear oscillatory neuron modeled by equation (1) with $p_{1}=0,4, \quad \mu_{1}=0,1$ and the frequency $\omega_{01}=0,2 \pi$, where the external signal $V_{1}(t)=v_{11}\left(t-t_{1}\right) \quad$ and the parameters $M_{1}=(3,4 \pi, 12,3,0)$, see (18), (20). This morphology depends on the way the external signal interacts with the own dynamics of the nonlinear oscillatory neuron.

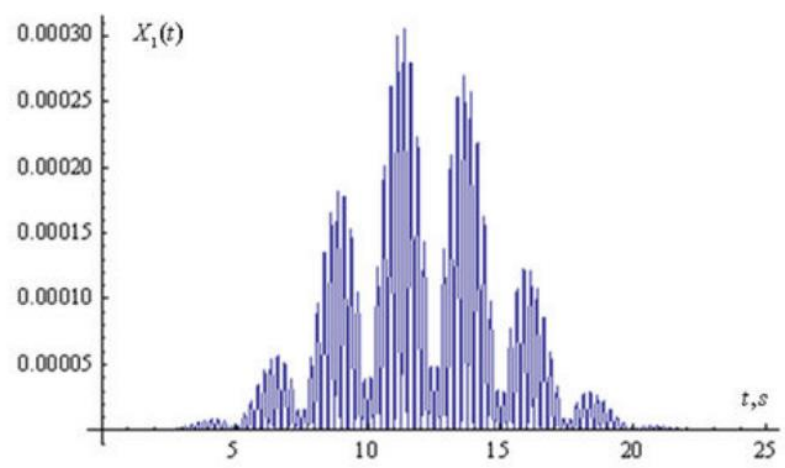

Fig.12. Morphology of the signal $X_{1}(t)$ at the output of the first oscillatory neuron, if the input signal $V_{1}(t)=v_{11}\left(t-t_{1}\right)$ and the parameters $M_{1}=(3,4 \pi, 12,3,0)$, see (19), (21). The signal $X_{1}(t)$ is the solution of system (1) with $N=1$ and the parameters $p_{1}=0,4$, $\mu_{1}=0,1$ and $\omega_{01}=0,2 \pi$.

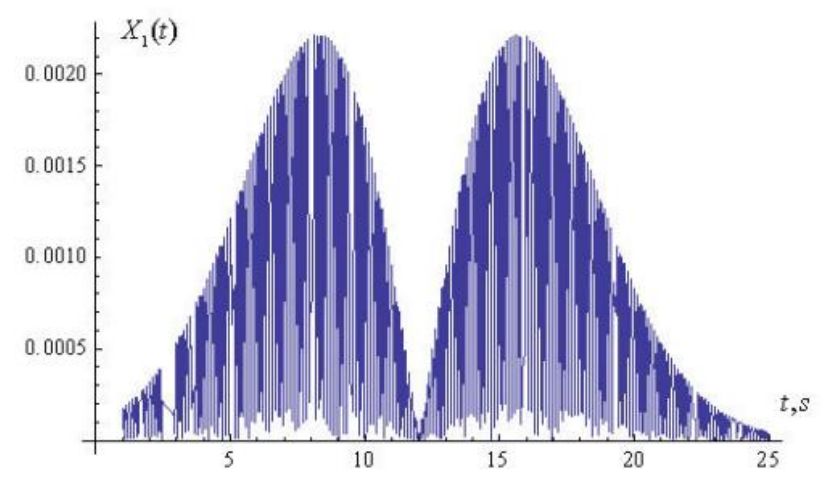

Fig.13. Morphology of the signal $X_{1}(t)$ at the output of the first oscillatory neuron, if the input signal $V_{1}(t)=v_{11}\left(t-t_{1}\right)$ and the parameters $M_{1}=(3,4 \pi, 12,3,0)$, see (19), (21). The signal $X_{1}(t)$ is the solution of system (1) with $N=1$ and the parameters $p_{1}=0,4$, $\mu_{1}=0,1$ and $\omega_{01}=4 \pi$.

Suppose now that the frequency of the single nonlinear oscillatory neuron from the last example is modified to $\omega_{01}=4 \pi$, which coincides with the frequency $\omega_{1}=4 \pi$ of the external signal $V_{1}(t)=v_{11}\left(t-t_{1}\right)$, see the list of parameters in Fig. 12 and (19), (21). Under this parameter 
regime, see Fig. 13, we observe a sharp amplitude rise of the output signal $X_{1}(t)$ compared to the output signal in Fig. 12. Thus, a resonance effect takes place if the own frequency of the single neuron coincides with the frequency of the external signal.

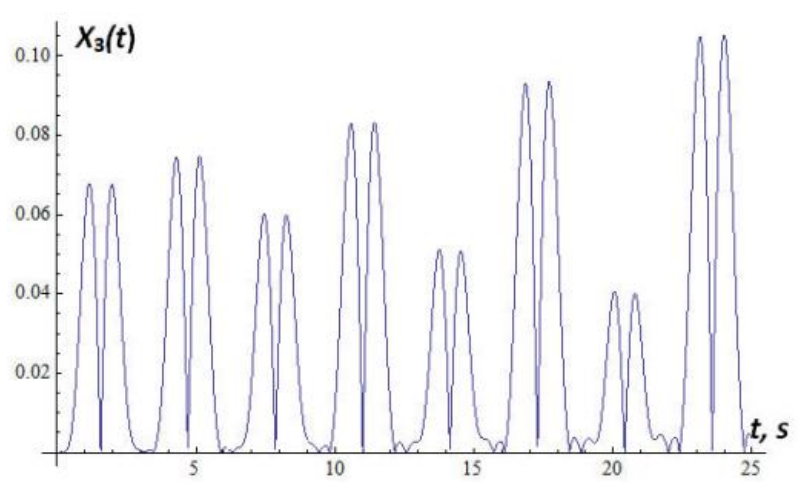

Fig.14. Morphology of the signal $X_{3}(t)$ at the output of the neural network with ring topology and two-sided connections in Fig. 3(b) modeled by system (3) with $N=3$ that consists of three nonlinear oscillatory neurons. The input is the harmonic signal $V_{1}=V_{0} \cos \tilde{\omega} t$,

$$
\begin{gathered}
V_{0}=1, \tilde{\omega}=3 \text { and the parameter values are } \omega_{01}=\omega_{02}=\omega_{03}=2, \\
p_{1}=0,4, p_{2}=0,3, p_{3}=0,2, \mu_{1}=0,1, \mu_{2}=0,2, \mu_{3}=0,4, \\
\lambda_{21}=\lambda_{13}=0,5, \lambda_{12}=\lambda_{23}=\lambda_{32}=0,4 .
\end{gathered}
$$

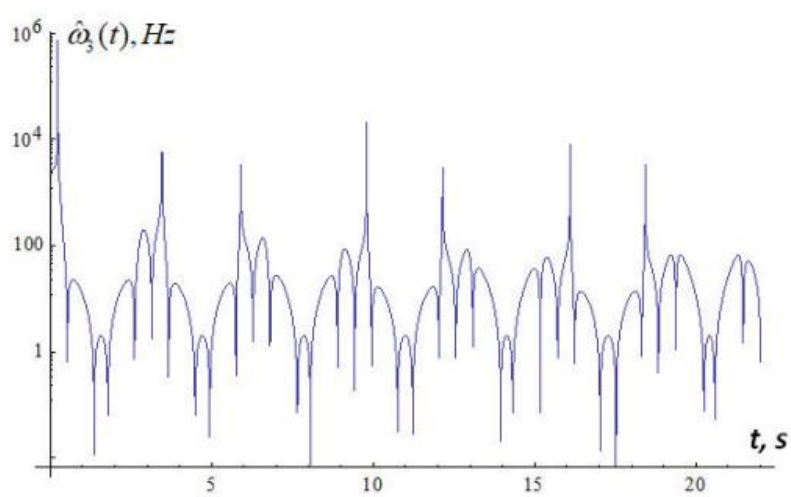

Fig.15. Frequency modulation of the output informational signal $X_{3}(t)$ plotted in Fig. 14, in semi-logarithmic scale.

Next, we consider the case where a harmonic signal arrives at the input of a three-neuron van der Pol type artificial nonlinear neural network with the morphology of a chain with two-sided connections (as in Fig. 2, but with two-sided connections). Wavelet analysis [25 - 27] of the morphology of this signal, see Fig. 14, shows that the structure of the output signal in this nonlinear network has a higher degree of stochasticity - with respect to the form, amplitude and the time-dependence of the instantaneous frequency of the bearing informational signal (Fig. 15) - compared to the signal at the output of a single neuron. This is related to the following feature of the three-neuron chain-like nonlinear-neuron network considered here: The structure (form, amplitude and frequency modulation) of the informational signal $X_{3}(t)$ at the output of the ring is influenced not only by the synaptic connections between the nonlinear oscillatory neurons, but also by the interaction between the own, internal dynamics of each neuron with the dynamics of the signal that arrives at that neuron.

To recover (decode) the incoming signal $V_{1}(t)$ from the output signal $X_{3}(t)$, one may plug in $X_{3}(t)$ in the third equation of system (3) with $N=3$ to find $V_{3}(t)=X_{2}(t)$, then plug in $X_{2}(t)$ into the second equation of the same system to find $V_{2}(t)=X_{1}(t)$ and, finally, plug in $X_{1}(t)$ into the first equation of the same system to obtain the bearing informational signal $V_{1}(t)$. Of course, in order to decode the output signal $X_{3}(t)$, one must know the parameters of system (3).

The results presented here suggest the following conjecture: the process of coding of the information by a single neuron or a nonlinear neural network can be considered in terms of the frequency modulation. Indeed, frequency modulation is known as one of the ways to transmit information. Therefore, it is expected that frequency modulation is one of the ways that neurons encode information.

\section{CONCLUSIONS}

Based on the frequency modulation approach, a nonlinear model of coding of information is presented. The model encodes incoming informational signals via a network of nonlinear oscillatory neurons with the topology of a chain or a ring. The neurons in the network are connected by synaptic links. The decoding of the signals at the output of the linear chain and circular chain networks is performed using a simple process of substitution of the output signal into a system of ODEs (note that it is not necessary to solve the system to decode the signal, only to substitute it into the equations of the system successively). The chain-like and the ring-like networks of nonlinear oscillatory neurons connected via synaptic links are described by nonlinear systems of ordinary differential equations, with quadratic nonlinearity of the unknown functions near the first derivative.

A method of solution of such systems of ODEs is proposed, based on the method of Krylov-BogolyubovMytropolsky and the method of subsequent approximations. It is observed that in both kinds of networks the nonlinear oscillatory neurons play the role of frequency modulators. That is, the neurons modulate the instantaneous frequency of the incoming nonstationary signal that arrives at their input.

It is shown that the structure of the signal at the output of the linear network (of nonlinear neurons) has a higher degree of stochasticity - with respect to the form, amplitude and the time dependence of the instantaneous frequency - compared to the morphology of the signal at the output of a single neuron.

Existence of resonance effects in chain-like networks is demonstrated, under the condition that the frequency of the incoming nonstationary signal equals the frequency of the own, internal dynamics of the neuron. 


\section{ACKNOWLEDGMENT}

This work was supported in part by the grant "Ontology based methods and means of construction of decision making support systems" (PI V. Lytvyn), Ministry of Education of Ukraine.

\section{REFERENCES}

[1] Sauer T. Reconstruction of dynamical systems from interspike intervals[J]. Phys. Rev. Lett., 1994, 72: 3811 3814.

[2] Marzantowicza W., Signerska J. On the interspikeintervals of periodically-driven integrate-and-fire models[J]. Journal of Mathematical Analysis and Applications, 2015, 423: 456 - 479 .

[3] O. N. Pavlova A. N. Pavlov. Improving the quality of extracting dynamics from interspike intervals via a resampling approach $[\mathrm{J}]$. Communications in Nonlinear Science and Numerical Simulation, 2018, 57: 221-230.

[4] Sangmin Song, Ji Ah Lee, Ilya Kiselev, Varun Iyengar, Josef G. Trapani, Nessy Tania. Mathematical Modeling and Analyses of Interspike-Intervals of Spontaneous Activity in Afferent Neurons of the Zebrafish Lateral-Line. Biophysical Journal, 2017, 112(3): 136a.

[5] Racicot D. M., Lonytin A., Interspike interval attractors from chaotically driven neuron models[J]. Physica D, 1997 , 104: $184-204$.

[6] Castro R., Sauer T. Correlation dimension of attractors through interspike intervals[J]. Phys. Rev. E 1997, 55: 287 $-290$.

[7] Sauer T. Nonlinear dynamics and time series. Eds C. Culter and D. Kaplan. Fields Institute Communications. American Mathemtical Society, 1997, RI 11: 63 - 75.

[8] Hegger R., Kantz H. Embedding of sequences of time intervals[J]. Europhys. Lett. 1997, 38: 267 - 272.

[9] Castro R., Sauer T. Chaotic Stochastic Resonance: NoiseEnhanced Reconstruction of Attractors[J]. Phys. Rev. Lett., 1997, 79: 1030 - 1033.

[10] Jinming Liu, Jian Mao, Bin Huang, Peiguo Liu. Chaos and reverse transitions in stochastic resonance[J]. Physics Letters A, 2018, 382: 3071-3078.

[11] Pavlov A. N., Hramov A. E., Koronovskij A. A., Sytnikova E. Ju., Makarov V. A., Ovchinnikov A. A. Wavelet analysis in neural dynamics[J]. Advances of Physical Sciences, 2012, 182(9): 905 - 939.

[12] Tuckwell H. C. Introduction to theoretical neurobiology. Cambridge University. Press, Cambridge, 1998.

[13] Janson N. B., Pavlov A. N., Neiman A. B., Anishchenko V. S. Reconstruction of dynamical and geometrical properties of chaotic attractors from threshold-crossing interspike intervals[J]. Phys. Rev. E, 1998, 58: R4 - R7.

[14] Pavlov A. N., Sosnovtseva O. V., Mosekilde E., Anishchenko V. S. Extracting dynamics from thresholdcrossing interspike intervals: Possibilities and limitations[J]. Phys. Rev. E, 2000, 61: 5033 - 5044.

[15] Pavlov A. N., Sosnovtseva O. V., Mosekilde E., Anishchenko V. S. Chaotic dynamics from interspike intervals[J]. Phys. Rev. E, 2001, 63: 036205.

[16] A. N. Pisarchik, G. Huerta-Cuellar, C. W. Kulp. Statistical analysis of symbolic dynamics in weakly coupled chaotic oscillators $[\mathrm{J}]$. Communications in Nonlinear Science and Numerical Simulation, 2018, 62: 134-145.
[17] John S., Bay and Hooshang Hemami. Modeling of a neural generator with coupled nonlinear oscillators. IEE Transactions biomedical engineering V, 1987, 34: 297 306.

[18] T. Shimoia, K. Mizutanib, D. Kojimaa, Y. Kitamurac, K. Hottaa, H. Ogawad, K. Oka. Identification of oscillatory firing neurons associated with locomotion in the earthworm through synapse imaging[J]. Neuroscience, 2014, 268: $149-158$

[19] Pavlov A. N., Pavlova O. N. Applications of wavelet analysis in the study of the structure of pointwise processes[J]. Letters to the Journal of Technical Physics, 2006, 32: 11 - 17 .

[20] Suhakov V. Y. Fundamentals of synergetics. Kyiv: Oberehy, 2001.

[21] Lytvyn V., Peleshchak I., Peleshchak R. The compression of the input images in neural network that use the diagonalization method for the matrices of synaptic weight connections[C]. 2nd International Conference on Advanced Information and Communication Technologies (AICT), 2017, 66 - 70

[22] Lytvyn V., Peleshchak I., Peleshchak R. Increasing the speed of detection and recognition of computer attacks in combined diagonalized neural networks[C]. 4th International Scientific-Practical Conference "Problems of infocommunications. Science and Technolohy", 2017, 152 -155 .

[23] Lytvyn V.V., Peleshchak I.R., Peleshchak R.M., Holoshchuk R.O., Detection of multispectral input images using nonlinear artificial neural networks[V]. 14th International Conference on Advanced Trends in Radioelectronics, Telecommunications and Computer Engineering, TCSET, 2018, $119-124$.

[24] Bogolyubov M. M., Yu. O. Mytropolsky. Asymptotical methods in the theory of nonlinear equations. Moscow: Nauka, 1992.

[25] Bozhokin S. V. Continuous wavelet transformation and exactly solvable model for non stationary signals $[\mathrm{J}]$. Journal of Technical Physics, 2012, 82: 8- 13.

[26] Grossmann A., Morlet J. Decomposition of Hardy functions into square integrable wavelets of constant shape[J]. SIAM. J. Math. Anal., 1984, 15: 723 - 736.

[27] Chui C. K., Wavelets: a mathematical tool for signal analysis (SIAM Monographs on Mathematical Modeling and Computation). Philadelphia: SIAM, 1997.

\section{Authors' Profiles}

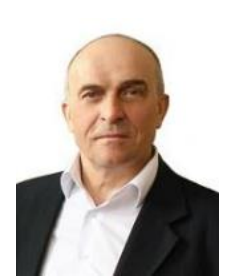

Roman Mykhaylovych Peleshchak graduated from Ivan Franko National University of Lviv, Ukraine, in 1980 with a major in radiophysics and electronics. In 2001 he received Doctor of Physical and Mathematical Sciences degree. Peleshchak's areas of expertise include theoretical physics, physics of intense nanoheterosystems with different dimensions, nonlinear neural networks and related encoding methods, pattern recognition, quantum dots in photodynamic therapy. He authored over 320 scientific papers. E-mail: rpeleshchak@ukr.net. 


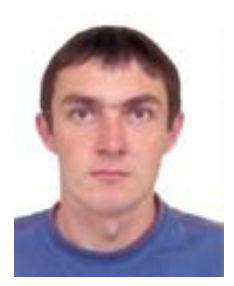

Vasyl Volodymyrovych Lytvyn is the chair of Information Systems and Networks Department of the Institute of Computer Science and Information Technology of Lviv Polytechnic National University, Ukraine. He graduated from Ivan Franko National University of Lviv, Ukraine, in 1997. In 2012 Lytvyn received Doctor of Technical Sciences degree. His doctoral dissertation is devoted to building decision support systems based on the ontological approach. Lytvyn's research interests include intelligent systems, machine learning, knowledge engineering and ontology construction. $\mathrm{He}$ published 170 scientific papers, 4 monographs and 5 textbooks. E-mail: vasyl.v.lytvyn@lpnu.ua.

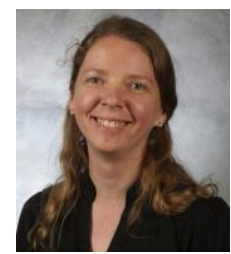

Oksana Bihun is an Assistant Professor at the Department of Mathematics of the University of Colorado, Colorado Springs, USA. She graduated from Ivan Franko National University of Lviv, Ukraine, in 2001 with a major in applied mathematics. Bihun received her Ph.D. in Mathematics from the University of Missouri, Columbia, USA, in 2009. Her research interests include integrable and solvable dynamical systems and their structures, orthogonal polynomials and special functions, numerical methods for solving differential equations and geometric analysis on manifolds. To date, Bihun's publication record consists of 28 papers in peer reviewed scientific journals, 23 abstracts or conference proceedings and 1 book chapter. E-mail: obihun@uccs.edu.

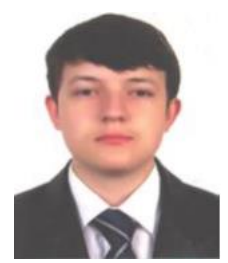

Ivan Romanovych Peleshchak graduated from Lviv Polytechnic National University, Ukraine, in 2017. E-mail: peleshchakivan@gmail.com.

How to cite this paper: Roman Peleshchak, Vasyl Lytvyn, Oksana Bihun, Ivan Peleshchak, "Structural Transformations of Incoming Signal by a Single Nonlinear Oscillatory Neuron or by an Artificial Nonlinear Neural Network", International Journal of Intelligent Systems and Applications(IJISA), Vol.11, No.8, pp.1-10, 2019. DOI: 10.5815/ijisa.2019.08.01 